\title{
Buckling of Shear Deformable Functionally Graded Orthotropic Cylindrical Shells under a Lateral Pressure
}

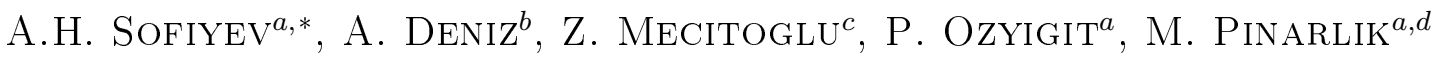 \\ ${ }^{a}$ Department of Civil Engineering, Engineering Faculty, \\ Suleyman Demirel University, Isparta, Turkey \\ ${ }^{b}$ Department of Mathematics of Usak University, Usak, Turkey \\ ${ }^{c}$ Aeronautics and Astronautics Faculty, Istanbul Technical University, Istanbul, Turkey \\ ${ }^{d}$ Department of Civil Engineering, Technology Faculty, Gazi University, Ankara, Turkey
}

In this study, the buckling of functionally graded $(\mathrm{FG})$ orthotropic cylindrical shells with shear deformation subjected to a lateral pressure is discussed. The stability and compatibility equations for FG orthotropic cylindrical shells on the basis of first order shear deformation theory (FOSDT) are derived. The expressions for non-dimensional critical lateral pressure on the basis of FOSDT and classical shell theory (CST) are obtained. The parametric studies are carried out to investigate the influences of shear deformation, orthotropy and heterogeneity on the non-dimensional critical lateral pressure.

DOI: 10.12693 /APhysPolA.127.907

PACS: 61.43.Bn; 62.20.mq

\section{Introduction}

In many practical applications, shell type structural elements have to work under radiation and high temperature environment which causes non-homogeneity in the material. However, the non-homogeneity of the materials stems from the effects of humidity, surface and thermal polishing processes and methods of production, which render the mechanical properties of materials, vary with space variables $[1,2]$. The shear deformation plays a significant role in the buckling behavior of shells composed of traditional and new generation composites. If the effect of shear deformation is not considered, it can lead to significant errors for the buckling loads of composite cylindrical shells. Thus, the shear deformable shell theory (SDST) becomes more interesting than the classical shell theory (CST) [3]. Due to the increased relevance of the FG orthotropic cylindrical shells in the design of aerospace structures, their buckling characteristics with account taken of combined effect of non-homogeneity and shear deformation has vital importance [4]. In this study, the buckling of shear deformable FG orthotropic cylindrical shells under a lateral pressure is investigated.

\section{Formulation of the problem}

Figure 1 shows the nomenclature of a circular cylindrical shell with radius $R$, length $L$ and thickness $h$. The cylindrical shell subjected to uniform lateral pressure. The origin of the coordinate system $(O x y z)$ is located at the end of the shell on the reference surface, in which

${ }^{*}$ corresponding author; e-mail: as of iyev@mmf .sdu.edu.tr $x$ and $y$ are in the axial and circumferential directions and $z$ is in the direction of the inward normal to the reference surface, respectively. Assume that the Young's moduli and shear moduli of the orthotropic materials are linear functions of the coordinate in the thickness direction [2]:

$$
\begin{aligned}
& E_{1}=E_{01}(1+\mu \bar{z}), E_{2}=E_{02}(1+\mu \bar{z}), \\
& G_{12}=G_{012}(1+\mu \bar{z}), G_{13}=G_{013}(1+\mu \bar{z}), \\
& G_{23}=G_{023}(1+\mu \bar{z}), \bar{z}=z / h,
\end{aligned}
$$

where $E_{01}$ and $E_{02}$ are Young's moduli of $H$ orthotropic material along $x$ and $y$ directions, respectively; $G_{012}, G_{013}, G_{023}$ are shear moduli which characterize angular changes between principal directions $x$ and $y, x$ and $z, y$ and $z$, respectively; $\mu$ is the variation coefficient of Young's moduli and shear moduli and satisfies $0 \leq \mu \leq 1$.

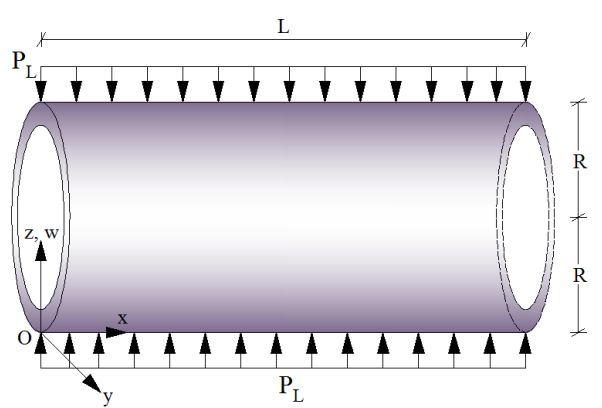

Fig. 1. Nomenclature and coordinate system of a cylindrical shell under a lateral pressure.

The equations relating the stresses to strains for shear deformable FG orthotropic cylindrical shells, in term of 
structural axes coordinates are given by the following matrix equations [5]:

$$
\begin{aligned}
& \left(\begin{array}{l}
\sigma_{1} \\
\sigma_{2} \\
\sigma_{12}
\end{array}\right)=\left[\begin{array}{ccc}
Q_{11}(\bar{z}) & Q_{12}(\bar{z}) & 0 \\
Q_{21}(\bar{z}) & Q_{22}(\bar{z}) & 0 \\
0 & 0 & Q_{66}(\bar{z})
\end{array}\right]\left(\begin{array}{l}
\varepsilon_{1} \\
\varepsilon_{2} \\
\varepsilon_{12}
\end{array}\right), \\
& \left(\begin{array}{c}
\sigma_{13} \\
\sigma_{23}
\end{array}\right)=\left[\begin{array}{cc}
Q_{55}(\bar{z}) & 0 \\
0 & Q_{44}(\bar{z})
\end{array}\right]\left(\begin{array}{l}
\varepsilon_{13} \\
\varepsilon_{23}
\end{array}\right)
\end{aligned}
$$

where $Q_{i j}(\bar{z})$ are the FG orthotropic stiffness which are related to engineering elastic constants.

The shear deformation shape function is distributed hyperbolic cosine manner through the shell thickness, i.e., $f(\bar{z})=\cosh (\bar{z})-\cos (1 / 2)[3]$. In accordance with the FOSDT, the strains at $\bar{z}$ distance from the reference surface of the FG orthotropic cylindrical shell are given as follows [3]:

$$
\begin{aligned}
& \varepsilon_{1}=\varepsilon_{1}^{0}-z w,_{x x}+I_{01} \phi,,_{x} ; \\
& \varepsilon_{2}=\varepsilon_{2}^{0}-z w,_{y y}+I_{02} \psi,,_{y} ; \\
& \varepsilon_{12}=\varepsilon_{12}^{0}-2 z w,_{x y}+I_{01} \phi,{ }_{y}+I_{02} \psi,{ }_{x}
\end{aligned}
$$

where a comma denotes partial differentiation with respect to the corresponding coordinates, $w$ is the displacement, $\phi$ and $\psi$ are the angles of rotation, $\varepsilon_{1}^{0}, \varepsilon_{2}^{0}, \varepsilon_{12}^{0}$ are strains on the reference surface and the following definitions apply:

$$
\begin{aligned}
I_{01} & =\int_{0}^{z} f(\bar{z}) / G_{13}(\bar{z}) d z, \\
I_{02} & =\int_{0}^{z} f(\bar{z}) / G_{23}(\bar{z}) d z .
\end{aligned}
$$

The force and moment resultants are defined as follows $[5]$ :

$$
\begin{aligned}
& \left(T_{1}, T_{2}, T_{12}, Q_{1}, Q_{2}\right)=\int_{-h / 2}^{h / 2}\left(\sigma_{1}, \sigma_{2}, \sigma_{12}, \sigma_{13}, \sigma_{23}\right) d z \\
& \left(M_{1}, M_{2}, M_{12}\right)=\int_{-h / 2}^{h / 2}\left(\sigma_{1}, \sigma_{2}, \sigma_{12}\right) z d z
\end{aligned}
$$

where $T_{1}$ and $T_{2}$ are normal forces; $T_{12}$ is the tangential force; $Q_{1}$ and $Q_{2}$ are shear forces; $M_{1}$ and $M_{2}$ are bending moments and $M_{12}$ is the torque moment.

The relations between the normal and tangential forces and Airy stress function are expressed as follows [2, 5]:

$$
\left(T_{1}, T_{12}, T_{2}\right)=h\left(\Phi,_{y y},-\Phi,_{x y}, \Phi,_{x x}\right)
$$

Substituting (3) into Eq. 2, then taking into account the resulting relations in Eq. 5, after some mathematical rearrangements, the relations found for normal and shear forces, moments and strains, and then considering these relations in the stability and compatibility equations [3] together with the relation (6), the following partial differential equations for FG orthotropic cylindrical shells in matrix form are obtained:

$$
\left[\begin{array}{llll}
L_{11} & L_{12} & L_{13} & L_{14} \\
L_{21} & L_{22} & L_{23} & L_{24} \\
L_{31} & L_{32} & L_{33} & L_{34} \\
L_{41} & L_{42} & L_{43} & L_{44}
\end{array}\right]\left[\begin{array}{c}
\Phi \\
w \\
\phi \\
\psi
\end{array}\right]=\left[\begin{array}{l}
0 \\
0 \\
0 \\
0
\end{array}\right]
$$

where $L_{i j}(i, j=1,2,3,4)$ are differential operators and are given in [2].

\section{Solution of basic equations}

The ends of a FG cylindrical shell are considered as simply supported, so that the solution can be described by [3]

$$
\begin{aligned}
& \Phi=C_{\varphi} \sin \left(m_{1} x\right) \sin \left(m_{2} y\right), \\
& w=C_{f} \sin \left(m_{1} x\right) \sin \left(m_{2} y\right), \\
& \phi=C_{\phi} \cos \left(m_{1} x\right) \sin \left(m_{2} y\right), \\
& \psi=C_{\psi} \sin \left(m_{1} x\right) \cos \left(m_{2} y\right)
\end{aligned}
$$

where $C_{\varphi}, C_{f}, C_{\phi}, C_{\psi}$ are unknown coefficients, $m_{1}=$ $m \pi / L, m_{2}=n / R$, in which, $m$ is the half wave number in axial direction and $n$ is the wave number in the circumferential direction. Substituting (8) into a set of equations (7), the following expression is obtained for $P_{\text {FOSDT }}^{\mathrm{Lcr}}$ of FG orthotropic cylindrical shell based on the FOSDT:

$$
P_{\mathrm{FOSDT}}^{\mathrm{Lcr}}=\frac{\lambda_{22} \lambda_{11}-\lambda_{12} \lambda_{21}}{\lambda_{11} m_{2}^{2} R E_{02}}
$$

where $\lambda_{i j}(i, j=1,2)$ are parameters depending on the material properties and shell characteristics. The expression of $P_{\mathrm{CST}}^{\mathrm{Lcr}}$ for FG orthotropic cylindrical shell based on the CST, as follows:

$$
\begin{aligned}
& P_{C S T}^{L c r}=\frac{1}{m_{2}^{2} R E_{02}}\left\{\left[c_{13} m_{1}^{4}+\left(c_{14}+2 c_{32}+c_{23}\right) m_{1}^{2} m_{2}^{2}+c_{24} m_{2}^{4}\right]+\left[m_{1}^{2} / R-c_{12} m_{1}^{4}-\left(c_{11}-2 c_{31}+c_{22}\right) m_{1}^{2} m_{2}^{2}-c_{21} m_{2}^{4}\right]\right. \\
& \left.\quad \times \frac{\left[m_{1}^{2} / R+b_{23} m_{1}^{4}+\left(b_{13}-b_{32}+b_{24}\right) m_{1}^{2} m_{2}^{2}+b_{14} m_{1}^{4}\right]}{b_{22} m_{1}^{4}+\left(b_{12}+b_{31}+b_{21}\right) m_{1}^{2} m_{2}^{2}+b_{11} m_{2}^{4}}\right\},
\end{aligned}
$$

where $b_{i j}, c_{i j}(i, j=1,2,3,4)$ are parameters depending on the material properties and shell characteristics.

\section{Numerical analysis}

A parametric study is intended to provide information on the buckling response of homogeneous and heterogeneous orthotropic cylindrical shells based on FOSDT and CST. Figure 2 shows the variation of $P_{\mathrm{FOSDT}}^{\mathrm{Lcr}}$ and $P_{\mathrm{CST}}^{\mathrm{Lcr}}$ for homogeneous and heterogeneous (linear function) cylindrical shells versus $R / h$ for $L / R=2$ and 3 . The material properties for homogeneous orthotropic materials are considered as $E_{01} / E_{02}=25, G_{012} / E_{02}=0.5$, $G_{023} / E_{02}=0.2, G_{013}=G_{012}, \nu_{12}=0.25$ [5]. It is observed that the values of $P_{\mathrm{FOSDT}}^{\mathrm{Lcr}}$ and $P_{\mathrm{CST}}^{\mathrm{Lcr}}$ for homo- 
geneous and FG orthotropic cylindrical shells decrease with increasing $R / h$. The effect of non-homogeneity on the values of $P_{\mathrm{FOSDT}}^{\mathrm{Lcr}}$ and $P_{\mathrm{CST}}^{\mathrm{LCr}}$ of the orthotropic cylindrical shell changes irregularly, as $R / h$ increases. In addition, this influence on the values of $P_{\mathrm{FOSDT}}^{\mathrm{Lcr}}$ is higher than $P_{\mathrm{CST}}^{\mathrm{Lcr}}$. For example, the influence of nonhomogeneity on $P_{\mathrm{FOSDT}}^{\mathrm{Lcr}}$ is $(\% 13.88 ; \% 13.94 ; \% 12.82$; $\% 13.33)$ and $(\% 13.36 ; \% 12.04 ; \% 11.54 ; \% 9.68)$ and on the values of $P_{\mathrm{CST}}^{\mathrm{Lcr}}$ is $(\% 6.84 ; \% 7.14 ; \% 6.33 ; \% 4.44)$ and (\%6.06; \%6.42; \%5.66; \%6.45) as $R / h=20 ; 30 ; 40 ; 50$ respectively, for $L / R=2$ and 3 , respectively. The difference between the values of $P_{\mathrm{FOSDT}}^{\mathrm{Lcr}}$ and $P_{\mathrm{CST}}^{\mathrm{Lcr}}$ for $\mathrm{FG}$ orthotropic shells is reasonable (about 10\%), whereas, this difference for homogeneous orthotropic shell is very slight. The effect of shear deformation on the $P_{\text {FOSDT }}^{\mathrm{Lcr}}$ irregularly decreases, as $R / h$ increases.

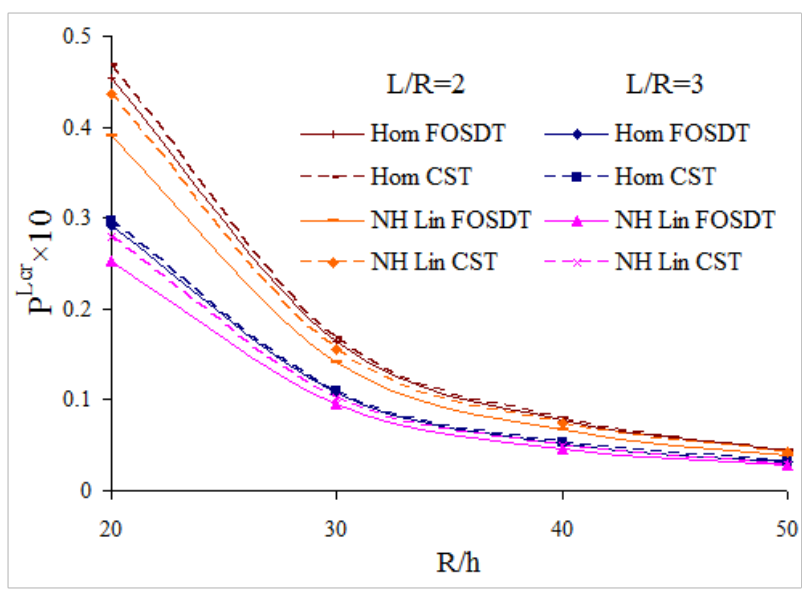

Fig. 2. Variations of $P_{\mathrm{FOSDT}}^{\mathrm{Lcr}}$ and $P_{\mathrm{CST}}^{\mathrm{Lcr}}$ for $\mathrm{H}$ and FG cylindrical shells versus $R / h$ for different $L / R$.

\section{Conclusions}

The buckling of shear deformable FG orthotropic cylindrical shells under a lateral pressure is investigated. The expressions for non-dimensional critical lateral pressure on the basis of FOSDT and CST are obtained. The numerical results support the following conclusions: The critical lateral pressures for homogeneous and FG orthotropic cylindrical shells decrease with increasing $R / h$. The effect of non-homogeneity on the critical lateral pressures changes irregularly, as $R / h$ increases. The influence of non-homogeneity on the values of $P$ Fos is higher than $P_{\mathrm{CST}}^{\mathrm{Lcr}}$. The difference between the values of $P_{\mathrm{FOSDT}}^{\mathrm{Lcr}}$ and $P$ CST for FG orthotropic shells is reasonable.

\section{Acknowledgments}

The authors thank Scientific and Technical Research Council of Turkey for the support of the project $113 \mathrm{M} 399$.

\section{References}

[1] J. Awrejcewicz and V.A. Krysko, Theory of nonhomogeneous shells in Chaos in Structural Mechanics Springer-Verlag, Berlin, 15 (2008).

[2] A.M. Najafov, A.H. Sofiyev and N. Kuruoglu, Meccanica 48, 829 (2013).

[3] T. Timarci, K.P. Soldatos, J. Sound Vibr. 187, 609 (1995).

[4] H. Matsunaga, Compos. Struct. 88, 519531 (2009).

[5] M.S. Qatu, Vibration of Laminated Shells and Plates, Elsevier, London 2004. 